\title{
Polarization of nucleons in the deuteron stripping reaction on nuclei
}

\author{
V. I. Kovalchuk \\ Department of Physics, Taras Shevchenko National University, Kiev 01033, Ukraine
}

\begin{abstract}
A general analytical expression has been obtained in the diffraction approximation for the polarization of nucleons arising in the deuteron stripping reaction on nuclei at intermediate energies of the incident particles. A tabulated density distribution of the target nucleus and a realistic wave function of the deuteron with correct asymptotic limits at large internucleon distances were used in the calculations. The nucleon-nucleus phase shifts were calculated in the Glauber formalism using the double folding potential. The calculated angular dependencies for the vector analyzing power $A_{y}$ of the reaction ${ }^{3} \mathrm{He}(\boldsymbol{d}, p){ }^{4} \mathrm{He}$ are found to be in satisfactory agreement with the corresponding experimental data.

PACS numbers: 24.10.Ht, 24.50.+g, 24.70.+s
\end{abstract}

\section{INTRODUCTION}

Nuclear reactions involving deuterons are widely used to examine the properties of the nuclear interaction and nuclear structure. The importance of such reactions in experimental nuclear physics is due both to the relative simplicity of obtaining monochromatic deuteron beams with precisely calibrated polarization and to the higher yield of $d A$-reactions in comparison with reactions induced by other particles. By virtue of the smallness of the binding energy of the deuteron, when a deuteron collides with a nucleus inelastic processes are most likely to occur: breakup of the deuteron in the Coulomb field of the target nucleus (mainly at low energies) and deuteron stripping, which occurs when one of the nucleons making up the deuteron is absorbed by the target and the other one is liberated as a reaction product. At intermediate energies the stripping reaction is the result of direct interaction (nucleon capture by the nucleus), and its differential cross section is characterized by a narrow peak at nucleon emission angles $\Theta \ll 1$.

A formalism for the inclusive deuteron stripping reaction at intermediate energies was first proposed by Serber [1] and was then developed further by Akhiezer and Sitenko [2, 3]. In [4] it was shown that the quintuple integral in the formula for the cross section of the reaction [3] can be calculated analytically if the integrand 
functions are expanded in a series over a Gaussian basis. In this approach, the scattering phase shifts were calculated in the Glauber formalism using the double folding potential [5] and tabulated distributions of the nucleon densities of the target nuclei [6], so that the final result (cross section) depends on a single parameter the normalization parameter of the imaginary part of the nucleon-nucleus potential. The present work considers the more complex problem of the polarization of the nucleon reaction products of deuteron stripping. The nature of the origin of such polarization, for example, on protons, corresponds to the different probability of absorption of a neutron for the two possible orientations of its spin [7]. Since the spins of the nucleons in a deuteron are parallel, the direction of the polarization of the escaping protons will be dictated by the orientation of the spin of the neutron, which has a greater probability of absorption. It is clear that the yield of polarized protons in the stripping reaction will be increased if the incident deuterons are polarized, and that the direction of their polarization will coincide with the direction of the protons arising during stripping.

\section{FORMALISM}

As our targets, we choose light and intermediate nuclei since for such nuclei and intermediate energies of the deuterons in this problem the Coulomb interaction can be neglected. The polarization of the nucleons produced by a stripping reaction involving unpolarized deuterons is expressed in terms of the density matrix $\rho$ as follows [8]:

$$
P=\operatorname{Tr}(\sigma \rho) / \operatorname{Tr} \rho
$$

where $\sigma$ is the nucleon spin operator (the Pauli matrices).

Let the deuteron move in the positive direction of the $z$-axis of a Cartesian coordinate system defined such that the $x y$-plane is the plane of the impact parameter. We denote the proton entering into the composition of the deuteron by the subscript 1 , and the neutron, by the subscript 2 . In the diffraction approximation, the general expression for the density matrix of the stripping reaction has the form [3]

$$
\rho\left(\boldsymbol{k}_{1}\right)=\int d \boldsymbol{b}_{2}\left(1-\left|1-\Omega_{2}\right|^{2}\right) a_{k_{1}}\left(\boldsymbol{r}_{2}\right) a_{\boldsymbol{k}_{1}}^{\dagger}\left(\boldsymbol{r}_{2}\right)
$$

where $\boldsymbol{b}_{2}$ is the impact parameter vector of the neutron and $\Omega_{2}$ is the corresponding profile function. The quantity

$$
a_{\boldsymbol{k}_{1}}\left(\boldsymbol{r}_{2}\right)=(2 \pi)^{-3 / 2} \int d \boldsymbol{r}_{1} \exp \left(-i \boldsymbol{k}_{1} \boldsymbol{r}_{1}\right)\left(1-\Omega_{1}\right) \varphi_{0}(\boldsymbol{r}), \quad \boldsymbol{r}=\boldsymbol{r}_{1}-\boldsymbol{r}_{2}
$$

is the probability amplitude that the proton will have momentum $\boldsymbol{k}_{1}$ and the neutron will be found at the point $\boldsymbol{r}_{2}$, and $\varphi_{0}(\boldsymbol{r})$ is the wave function of the deuteron. 
The neutron profile function $\Omega_{2}$ in expression (2) contains only a radial part, i.e., $\Omega_{2}\left(b_{2}\right)=\omega_{2}\left(b_{2}\right)$, but the proton profile function also depends on the spin [9]

$$
\Omega_{1}\left(\boldsymbol{r}_{1}\right)=\omega_{1}\left(b_{1}\right)\left\{1+\gamma_{1} \exp \left(i \delta_{1}\right) \sigma\left((\boldsymbol{k} / 2) \times \partial / \partial \boldsymbol{r}_{1}\right)\right\},
$$

Here $b_{1}, \gamma_{1}$, and $\delta_{1}$ are, respectively, the impact parameter, a constant, and the phase shift of the spin-orbit interaction of the proton with the nucleus, and $\boldsymbol{k}$ is the momentum vector of the incident deuteron.

If Gaussian functions stand under the integral sign in Eq.(2), then the density matrix can be calculated in explicit form. Without loss of generality, we expand the functions $\omega_{i}\left(b_{i}\right) \quad(i=1,2)$ and $\varphi_{0}(\boldsymbol{r})$ in a Gaussian basis:

$$
\omega_{i}\left(b_{i}\right)=\sum_{j=1}^{N} \alpha_{i j} \exp \left(-b_{i}^{2} / \beta_{i j}\right), \quad \varphi_{0}(\boldsymbol{r})=\sum_{j=1}^{N} c_{j} \exp \left(-d_{j}\left|\boldsymbol{r}_{1}-\boldsymbol{r}_{2}\right|^{2}\right),
$$

where $\beta_{i j}=R_{r m s}^{2} / j$, and $R_{r m s}$ is the root-mean-square radius of the target nucleus.

A analytical integration in Eq.(2) with the above functions (formulas (5)) substituted in formulas (3) and (44), followed by calculation of the traces of the matrices in the numerator and the denominator of formula (1), yields the expressions $\operatorname{Tr}\left(\sigma \rho\left(\boldsymbol{k}_{1}\right)\right)=\boldsymbol{G}\left(\boldsymbol{k}_{1}\right)$ and $\operatorname{Tr}\left(\rho\left(\boldsymbol{k}_{1}\right)\right)=H\left(\boldsymbol{k}_{1}\right)$ (see Appendix A). Thus, the polarization of the proton is given by the formula

$$
\boldsymbol{P}\left(\boldsymbol{k}_{1}\right)=G\left(\boldsymbol{k}_{1}\right)\left(\boldsymbol{p}_{1} \times \boldsymbol{k}_{1}\right) / H\left(\boldsymbol{k}_{1}\right),
$$

where $\boldsymbol{p}_{1}$ is the transverse component of the momentum $\boldsymbol{k}_{1}=\left\{\boldsymbol{p}_{1},(\boldsymbol{k} / k) k_{1 z}\right\}$. The values of the components $p_{1}$ and $k_{1 z}$ of the vector $k_{1}$ are functions of the energy of the proton $T_{1}$ and its emission angle $\Theta_{1}$ in the laboratory coordinate system [3]:

$$
p_{1}=\left(k / 2+k_{1 z}\right) \tan \Theta_{1}, \quad k_{1 z}=\sqrt{m / T}\left(T_{1}-T / 2\right) .
$$

Here $m$ is the nucleon mass and $T$ is the initial energy of the deuteron.

Note that the denominator in expression (6) is determined by the doubly differential cross section of the stripping reaction (with respect to the emission angle of the nucleon and its energy) [3].

In order to find the angular distribution of the polarization, the functions $G\left(\boldsymbol{k}_{1}\right)$ and $H\left(\boldsymbol{k}_{1}\right)$ in expression (6) must be integrated with respect to the $z$-component of the vector $\boldsymbol{k}_{1}\left[3\right.$. Expressing $\boldsymbol{k}_{1}$ in terms of the components of the corresponding cylindrical coordinate system and taking relations (7) into account, we obtain

$$
P\left(\Theta_{1}\right)=\int_{-\infty}^{\infty}\left(k / 2+k_{1 z}\right)^{2} G\left(p_{1}, k_{1 z}\right) d k_{1 z} / \int_{-\infty}^{\infty}\left(k / 2+k_{1 z}\right)^{2} H\left(p_{1}, k_{1 z}\right) d k_{1 z} .
$$

To transform the angle $\Theta_{1}$ into the corresponding quantity in the center-of-mass system (c.m.s.), we use the formulas of relativistic kinematics given in [10]. 


\section{RESULTS AND DISCUSSION. CONCLUSIONS}

Current experiments measure, as a rule, not the polarization of the particles, rather the analyzing power of the reaction [11. These quantities are interrelated: thus, for example, for a stripping reaction with polarized deuterons, the vector analyzing power of the reaction $A_{y}=3 P$ [7, 11]. The formalism expounded in the present paper was used to describe the polarization observables $A_{y}$ of the reaction ${ }^{3} \mathrm{He}(\boldsymbol{d}, p){ }^{4} \mathrm{He}$ for deuteron energies $T=140$, 200, and $270 \mathrm{MeV}$ [12].

The values of the parameters $\alpha_{i j}$ in formulas (5) were calculated using nucleonnucleus phase shifts in the Glauber formalism [4, 5] and the experimentally obtained distribution of the nucleon density of the ${ }^{3} \mathrm{He}$ nucleus [6] (see Appendix B). To find the parameters $c_{j}$ and $d_{j}$ of the deuteron wave function (formulas (5)), the variational problem in the basis $N=10$ of Gaussian functions with the K2 triplet nucleon-nucleon potential from [13] was solved separately. This wave function has the correct asymptotic limits at large internucleon distances; moreover, it reproduces with a high degree of accuracy the experimental values of the binding energy of the deuteron and its root-mean-square radius.

Figure 1 plots the calculated angular dependencies of the vector analyzing power of the deuteron stripping reaction on ${ }^{3} \mathrm{He}$ nuclei for $T=140,200$, and $270 \mathrm{MeV}$. The values of the parameters of the spin-orbit interaction $\left(\gamma_{1}, \delta_{1}\right)$ were $(0.143,0.143)$ for $T=140 \mathrm{MeV},(0.141,0.117)$ for $T=200 \mathrm{MeV}$, and $(0.139,0.106)$ for $T=270 \mathrm{MeV}$. The normalization constant of the imaginary part of the double folding potential, $N_{I}$ (formula (B.6), Appendix B), was equal to unity in all cases.

The dash-dot curves in Fig. 1 correspond to the results of calculations of $A_{y}$ for the reaction of elastic $p d$-scattering, performed in [12] on the basis of the formalism of Faddeev equations with the realistic $N N$-potential AV18.

From an analysis of the behavior of the curves in the figure it follows that both dependencies qualitatively reproduce the behavior of the experimental data, and the curves have a minimum at the point at which the polarization changes sign.

Thus, the formalism presented in this paper for calculating the polarization of nucleons arising in the deuteron stripping reaction at intermediate energies allows at least a qualitative description of the corresponding experiments. The method is based on the use of Gaussian expansions of the distributions of the nucleon density of the target nuclei and the deuteron wave function, which made it possible to calculate the integrals analytically and to calculate the density matrix of the reaction and the mean value of the spin of the escaping nucleon in explicit form.

An inadequacy of the method is the relatively narrow range of angles in which agreement with experiment is obtained (due to limitations of the diffraction nuclear model [3]). In this work we also did not take into account the $D$-component in the 

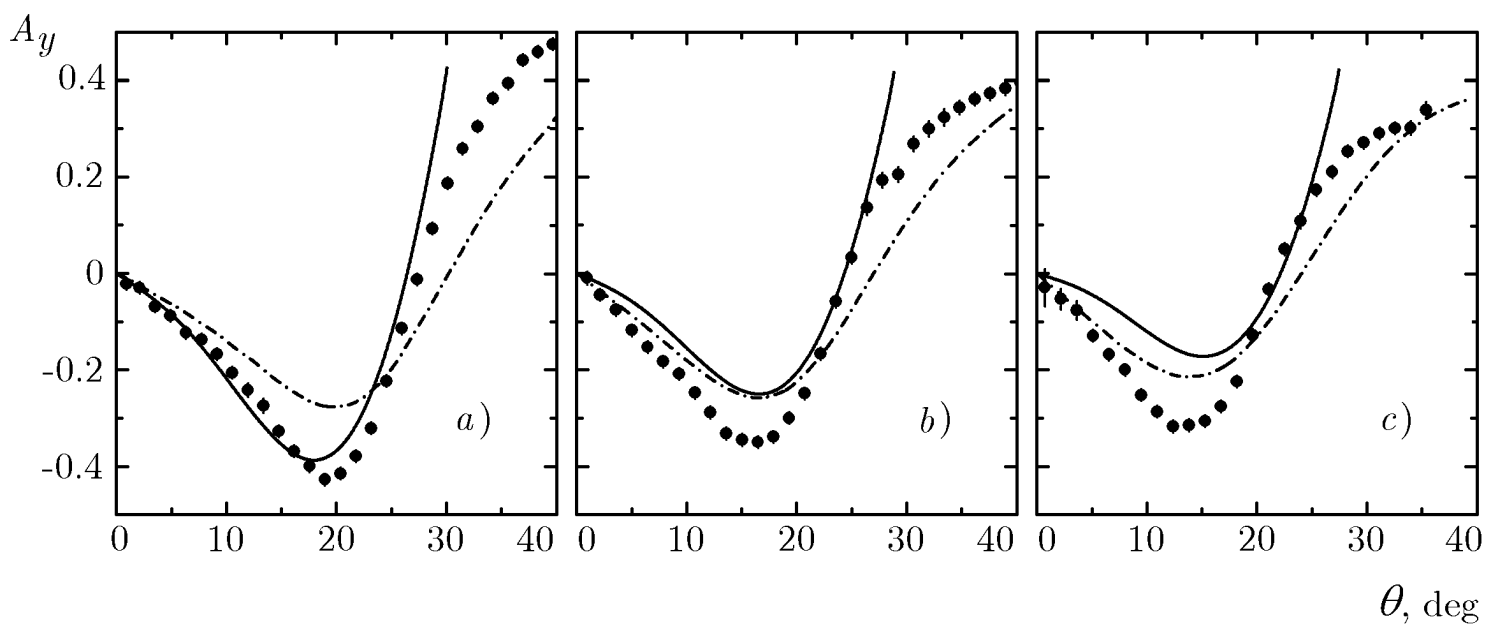

Figure 1: Vector analyzing power $A_{y}$ of the reaction ${ }^{3} \mathrm{He}(\boldsymbol{d}, p)^{4} \mathrm{He}$ as a function of the proton emission angle $\theta$ in the c.m.s. for incident deuteron energies $T=140(a), 200(b)$, and $270 \mathrm{MeV}(c)$. The curves are explained in the text. Experimental data from [12].

deuteron wave function although the expected correction to the vector analyzing power in this case would have amounted to several percent of the calculated value.

Overall, the proposed method can be used to analyze the corresponding experiments and determine the values of the parameters of the spin-orbit interaction and their energy dependence. It should be noted that the general formulas obtained in this work can be used to describe the cross sections and polarizations in inclusive processes not only of stripping, but also of breakup of deuterons, and also of light and heavy ions [14. In these cases, both the angular and the energy distributions of the indicated quantities can be calculated (in the latter case, the expressions for $G$ and $H$ appearing in expression (6) must be integrated over the perpendicular components of the momentum vector of the incident particle).

\section{Appendix A Traces of the density matrix $\rho$ and the products $\sigma \rho$}

We present the result of calculation of the traces of the matrices in the numerator and the denominator of expression (11) after analytical integration in expression (2) with the functions assigned by formulas (5).

The numerator in expression (11) has the form

$$
\operatorname{Tr}\left(\sigma \rho\left(\boldsymbol{k}_{1}\right)\right)=\boldsymbol{G}\left(\boldsymbol{k}_{1}\right)=G\left(\boldsymbol{k}_{1}\right)\left(\boldsymbol{p}_{1} \times \boldsymbol{k}_{1}\right) .
$$

Here $\boldsymbol{p}_{1}$ is the transverse component of the momentum of the incident nucleon: 
$\boldsymbol{k}_{1}=\left\{\boldsymbol{p}_{1}, \boldsymbol{k}_{1 z}\right\}$, and the quantity $G\left(\boldsymbol{k}_{1}\right)$ is defined as

$$
G\left(\boldsymbol{k}_{1}\right)=\gamma_{1} \sin \delta_{1} \sum_{s=1}^{N} \sum_{q=1}^{N} c_{s} c_{q} Z\left(\lambda, p_{1}, k_{1 z}\right)
$$

where

$$
\begin{gathered}
Z\left(\lambda, p_{1}, k_{1 z}\right)=2 \lambda t\left(\lambda, k_{1 z}\right)\left(4 z^{(1)}\left(\lambda, p_{1}\right)-z^{(2)}\left(\lambda, p_{1}\right)\right) \\
z^{(1)}=z_{11}-z_{12} \\
z^{(2)}=4 z_{21}+z_{22} .
\end{gathered}
$$

The denominator in expression (1) is equal to

$$
\operatorname{Tr} \rho\left(\boldsymbol{k}_{1}\right)=H\left(\boldsymbol{k}_{1}\right)=H_{0}\left(\boldsymbol{k}_{1}\right)+\gamma_{1}^{2} H_{\mathrm{so}}\left(\boldsymbol{k}_{1}\right),
$$

where

$$
\begin{gathered}
H_{0}\left(\boldsymbol{k}_{1}\right)=\sum_{s=1}^{N} \sum_{q=1}^{N} c_{s} c_{q} Y\left(\lambda, p_{1}, k_{1 z}\right), \\
Y\left(\lambda, p_{1}, k_{1 z}\right)=t\left(\lambda, k_{1 z}\right)\left(y^{(1)}\left(\lambda, p_{1}\right)-y^{(2)}\left(\lambda, p_{1}\right)\right), \\
y^{(1)}=4\left(y_{11}-2 y_{12}+y_{13}\right), \\
y^{(2)}=y_{21}-4 y_{22}+y_{23} ; \\
H_{\mathrm{so}}\left(\boldsymbol{k}_{1}\right)=\sum_{s=1}^{N} \sum_{q=1}^{N} c_{s} c_{q} W\left(\lambda, p_{1}, k_{1 z}\right), \\
w^{(1)}=\left(\lambda p_{1}, k_{1 z}\right)^{2} w_{11}+2\left(2 k_{1 z}^{2}+p_{1}^{2}\right) w_{12}, \\
w^{(2)}=\left(\lambda p_{1} k_{1 z}\right)^{2} w_{21}+\left(2 k_{1 z}^{2}+p_{1}^{2}\right) w_{22} .
\end{gathered}
$$

The quantities on the right-hand side of expressions (A.4), (A.5), (A.9), (A.10), (A.13), and (A.14) are defined as follows:

$$
\begin{gathered}
z_{11}=\sum_{i=1}^{N} \sum_{j=1}^{N} \frac{\alpha_{1 i} \beta_{1 i} \alpha_{2 j} \beta_{2 j}}{\left(\lambda+\beta_{1 i}+\beta_{2 j}\right)^{2}} \exp \left(-\frac{\lambda+2 \beta_{1 i}+2 \beta_{2 j}}{\lambda+\beta_{1 i}+\beta_{2 j}} \frac{\lambda p_{1}^{2}}{4}\right), \\
z_{12}=\sum_{i=1}^{N} \sum_{j=1}^{N} \sum_{l=1}^{N} \frac{\alpha_{1 i} \beta_{1 i} \alpha_{1 j} \beta_{1 j} \alpha_{2 l} \beta_{2 l}}{\left(\lambda+\beta_{1 i j}\right)^{2}\left(\lambda+\beta_{1 i j}+2 \beta_{2 l}\right)} \exp \left(-\frac{\beta_{1 i j}}{\lambda+\beta_{1 i j}} \frac{\lambda p_{1}^{2}}{2}\right), \\
z_{21}=\sum_{i=1}^{N} \sum_{j=1}^{N} \sum_{l=1}^{N} \frac{\alpha_{1 i} \beta_{1 i} \alpha_{2 j} \alpha_{2 l} \beta_{2 j l}}{\left(2 \lambda+2 \beta_{1 i}+\beta_{2 j l}\right)^{2}} \exp \left(-\frac{\lambda+2 \beta_{1 i}+\beta_{2 j l}}{2 \lambda+2 \beta_{1 i}+\beta_{2 j l}} \frac{\lambda p_{1}^{2}}{2}\right),
\end{gathered}
$$




$$
\begin{aligned}
& z_{22}=\sum_{i=1}^{N} \sum_{j=1}^{N} \sum_{l=1}^{N} \sum_{n=1}^{N} \frac{\alpha_{1 i} \beta_{1 i} \alpha_{1 j} \beta_{1 j} \alpha_{2 l} \alpha_{2 n} \beta_{2 l n}}{\left(\lambda+\beta_{1 i j}\right)^{2}\left(\lambda+\beta_{1 i j}+\beta_{2 l n}\right)} \exp \left(-\frac{\beta_{1 i j}}{\lambda+\beta_{1 i j}} \frac{\lambda p_{1}^{2}}{2}\right) ; \\
& y_{11}=\exp \left(-\frac{\lambda p_{1}^{2}}{2}\right) \sum_{i=1}^{N} \alpha_{2 i} \beta_{2 i} \\
& y_{12}=\sum_{i=1}^{N} \sum_{j=1}^{N} \frac{\alpha_{1 i} \beta_{1 i} \alpha_{2 j} \beta_{2 j}}{\lambda+\beta_{1 i}+\beta_{2 j}} \exp \left(-\frac{\lambda+2 \beta_{1 i}+2 \beta_{2 j}}{\lambda+\beta_{1 i}+\beta_{2 j}} \frac{\lambda p_{1}^{2}}{4}\right), \\
& y_{13}=\sum_{i=1}^{N} \sum_{j=1}^{N} \sum_{l=1}^{N} \frac{\alpha_{1 i} \beta_{1 i} \alpha_{1 j} \beta_{1 j} \alpha_{2 l} \beta_{2 l}}{\left(\lambda+\beta_{1 i j}\right)\left(\lambda+\beta_{1 i j}+2 \beta_{2 l}\right)} \exp \left(-\frac{\beta_{1 i j}}{\lambda+\beta_{1 i j}} \frac{\lambda p_{1}^{2}}{2}\right), \\
& y_{21}=\exp \left(-\frac{\lambda p_{1}^{2}}{2}\right) \sum_{i=1}^{N} \sum_{j=1}^{N} \alpha_{2 i} \beta_{2 i} \beta_{2 i j} \\
& y_{22}=\sum_{i=1}^{N} \sum_{j=1}^{N} \sum_{l=1}^{N} \frac{\alpha_{1 i} \beta_{1 i} \alpha_{2 j} \beta_{2 j} \beta_{2 j l}}{2 \lambda+2 \beta_{1 i}+\beta_{2 j l}} \exp \left(-\frac{\lambda+2 \beta_{1 i}+\beta_{2 j l}}{2 \lambda+2 \beta_{1 i}+\beta_{2 j l}} \frac{\lambda p_{1}^{2}}{2}\right),
\end{aligned}
$$

$$
y_{23}=\sum_{i=1}^{N} \sum_{j=1}^{N} \sum_{l=1}^{N} \sum_{n=1}^{N} \frac{a_{1 i} \beta_{1 i} a_{1 j} \beta_{1 j} a_{2 l} \beta_{2 l} \beta_{2 l n}}{\left(\lambda+\beta_{1 i j}\right)\left(\lambda+\beta_{1 i j}+\beta_{2 l n}\right)} \exp \left(-\frac{\beta_{1 i j}}{\lambda+\beta_{1 i j}} \frac{\lambda p_{1}^{2}}{2}\right)
$$

$$
w_{11}=\sum_{i=1}^{N} \sum_{j=1}^{N} \sum_{l=1}^{N} \frac{\alpha_{1 i} \beta_{1 i} \alpha_{1 j} \beta_{1 j} \alpha_{2 l} \beta_{2 l}}{\left(\lambda+\beta_{1 i j}\right)^{3}\left(\lambda+\beta_{1 i j}+2 \beta_{2 l}\right)} \exp \left(-\frac{\beta_{1 i j}}{\lambda+\beta_{1 i j}} \frac{\lambda p_{1}^{2}}{2}\right)
$$

$$
w_{12}=\sum_{i=1}^{N} \sum_{j=1}^{N} \sum_{l=1}^{N} \frac{\alpha_{1 i} \beta_{1 i} \alpha_{1 j} \beta_{1 j} \alpha_{2 l} \beta_{2 l}^{2}}{\left(\lambda+\beta_{1 i j}\right)^{2}\left(\lambda+\beta_{1 i j}+2 \beta_{2 l}\right)^{2}} \exp \left(-\frac{\beta_{1 i j}}{\lambda+\beta_{1 i j}} \frac{\lambda p_{1}^{2}}{2}\right),
$$

$$
w_{21}=\sum_{i=1}^{N} \sum_{j=1}^{N} \sum_{l=1}^{N} \sum_{n=1}^{N} \frac{\alpha_{1 i} \beta_{1 i} \alpha_{1 j} \beta_{1 j} \alpha_{2 l} \alpha_{2 n} \beta_{2 l n}}{\left(\lambda+\beta_{1 i j}\right)^{3}\left(\lambda+\beta_{1 i j}+\beta_{2 l n}\right)} \exp \left(-\frac{\beta_{1 i j}}{\lambda+\beta_{1 i j}} \frac{\lambda p_{1}^{2}}{2}\right),
$$




$$
w_{22}=\sum_{i=1}^{N} \sum_{j=1}^{N} \sum_{l=1}^{N} \sum_{n=1}^{N} \frac{\alpha_{1 i} \beta_{1 i} \alpha_{1 j} \beta_{1 j} \alpha_{2 l} \alpha_{2 n} \beta_{2 l n}^{2}}{\left(\lambda+\beta_{1 i j}\right)^{2}\left(\lambda+\beta_{1 i j}+\beta_{2 l n}\right)^{2}} \exp \left(-\frac{\beta_{1 i j}}{\lambda+\beta_{1 i j}} \frac{\lambda p_{1}^{2}}{2}\right) .
$$

In formulas (A.2), (A.7), and (A.11) $\lambda=2 /\left(d_{s}+d_{q}\right)$. The function $t\left(\lambda, k_{1 z}\right)$ in formulas (A.3), (A.8), and (A.12) has the form

$$
t\left(\lambda, k_{1 z}\right)=\pi^{4} \lambda^{3} \exp \left(-\frac{\lambda k_{1 z}^{2}}{2}\right) .
$$

Moreover,

$$
\beta_{i j l}=2 \beta_{i j} \beta_{i l} /\left(\beta_{i j}+\beta_{i l}\right), \quad(i=1,2 ; j, l=\overline{1, N}) .
$$

\section{Appendix B Calculation of the profile functions}

The radial parts of the nucleon-nucleus profile functions were calculated in the eikonal approximation

$$
\omega_{i}\left(b_{i}\right)=1-\exp \left[-\phi_{i}\left(b_{i}\right)\right], \quad i=1,2 ;
$$

where

$$
\phi_{i}\left(b_{i}\right)=-\frac{1}{v} \int_{-\infty}^{\infty} d z W\left(\sqrt{b_{i}^{2}+z^{2}}\right)
$$

is the scattering phase shift, $v$ is the velocity of the incident nucleon, and $W(r)$ is the imaginary part of the nucleon-nucleus potential.

Within the framework of the double folding model, the eikonal phase shift can be calculated by the method described in [5]. Let the density distribution of nuclear matter in a nucleon $\left(\rho_{i}(r)\right)$ and the amplitude of the nucleon-nucleus interaction in the plane of the impact parameter $(f(b))$ be described by Gaussian functions

$$
\begin{gathered}
\rho_{i}(r)=\rho_{i}(0) \exp \left(-r^{2} / a_{i}^{2}\right), \\
f(b)=\left(\pi r_{0}^{2}\right)^{-1} \exp \left(-b^{2} / r_{0}^{2}\right),
\end{gathered}
$$

where $\rho_{i}(0)=\left(a_{i} \sqrt{\pi}\right)^{-3}, a_{i}^{2}=r_{0}^{2}=2 r_{N N}^{2} / 3$, and $r_{N N}^{2} \cong 0.65 \mathrm{fm}^{2}$ is the meansquare radius of $N N$-interaction. If the density distribution of the target nucleus (the tabulated distribution [6] or the model distribution) is expanded in a series in the Gaussian basis

$$
\rho_{T}(r)=\sum_{j=1}^{N} \rho_{T j} \exp \left(-r^{2} / a_{T j}^{2}\right), \quad a_{T j}^{2}=R_{r m s}^{2} / j,
$$

where $R_{r m s}$ is the root-mean-square radius of the nucleus, then the formula for the eikonal phase shift [5] can be generalized [4]:

$$
\phi_{i}\left(b_{i}\right)=N_{W} \sqrt{\pi} \bar{\sigma}_{N N} \sum_{j=1}^{N} \frac{\rho_{T j} a_{T j}^{3}}{a_{T j}^{2}+2 r_{0}^{2}} \exp \left(-\frac{b_{i}^{2}}{a_{T j}^{2}+2 r_{0}^{2}}\right) .
$$


Here $N_{W}$ is the normalization factor for the imaginary part of the double folding

potential and $\bar{\sigma}_{N N}$ is the isotopically averaged cross section of the nucleon-nucleus interaction.

Formula (B.6) was used directly in the calculations of the profile functions (Eqs. (B.1)), which were then expanded in the Gaussian basis (formulas (5D)).

\section{REFERENCES}

1. R. Serber, Phys. Rev. 72, 1008 (1947).

2. A. I. Akhiezer and A. G. Sitenko, Sov. Phys. JETP 6, 799 (1958).

3. A. G. Sitenko, Theory of Nuclear Reactions (World Scientific, Singapore, 1990).

4. V. I. Kovalchuk, Nucl. Phys. A 937, 59 (2015).

5. S. K. Charagi and S. K. Gupta, Phys. Rev. C 41, 1610 (1990).

6. H. De Vries, C. W. De Jager, and C. De Vries, At. Data Nucl. Data Tabl. 36, 495 (1987).

7. G. L. Vysotskii and A. G. Sitenko, Sov. Phys. JETP 9, 812 (1959).

8. A. G. Sitenko, Scattering Theory: Lecture Notes (Springer-Verlag Berlin Heidelberg, 2012).

9. A. G. Sitenko, Nucl. Phys. 9, 412 (1958/59).

10. A. M. Baldin, W. I. Goldanskij, and I. L. Rosental, Kinematik der Kernreaktionen (Akademie-Verlag, Berlin, 1963).

11. R. W. Nielsen, Nuclear Reaction: Mechanism and Spectroscopy (Griffith University, Australia, 2011).

12. H. Kamada, T. Uesaka, W. Glöckle, et al. Prog. Theor. Phys. Lett. 104, 703 (2000).

13. D. V. Piatnytskyi and I. V. Simenog, Ukr. J. Phys. 53, 629 (2008).

14. H. Utsunomiya, Phys. Rev. C 32, 849 (1985). 